\title{
Hydration and proton conductivity of ionomers: the model case of sulfonated aromatic polymers
}

\author{
Philippe Knauth $^{\mathbf{1}}{ }^{*}$ and Maria Luisa Di Vona ${ }^{2}$ \\ ${ }^{1}$ Madirel (UMR 7246), CNRS, Aix Marseille Université, Marseille, France \\ ${ }^{2}$ Dipartimento di Scienze e Tecnologie Chimiche, Università di Roma Tor Vergata, Roma, Italy
}

\section{Edited by:}

Venkataraman Thangadurai, University of Calgary, Canada

\section{Reviewed by:}

Hongying Hou, Kunming University of Science and Technology, China

Kunal Karan, University of Calgary,

Canada

*Correspondence:

Philippe Knauth, Aix Marseille

Université, Campus St Jérôme,

13397 Marseille, France

e-mail: philippe.knauth@univ-amu.fr
The hydration of proton-conducting ionomers is described in terms of a simplified model, where only osmotic and elastic contributions to the Gibbs free energy of hydration are considered. Although only two physically meaningful parameters are used - the deformation parameter, inversely proportional to the elastic modulus of the ionomer, and the free volume parameter - simulated hydration isotherms are in good agreement with the experiment. The proton mobility $u$ inside the electrolyte solution of the ionomer is calculated from the proton conductivity determined at various hydration numbers. Its variation with the proton concentration $c$ reveals the percolation threshold of hydrated nanometric channels and the tortuosity of the membrane. Above the percolation threshold, a power law $u \sim c^{-3}$ is observed, in agreement with the "universal" law for 3-dimensional percolation. The proton conductivity $\sigma$ shows at $100^{\circ} \mathrm{C}$ a maximum of $0.2 \mathrm{~S} / \mathrm{cm}$ at a hydration number 90. The $\sigma=f(c)$ plot allows to predict, which hydration conditions are necessary for a desired area specific resistance.

Keywords: SPEEK, SPPSU, proton mobility, hydration number, water uptake, elastic modulus

\section{INTRODUCTION}

Polymer electrolytes are a fascinating class of materials with many promising applications, especially in the field of energy and environment (Armand, 1994; Meyer, 1998; Fergus, 2010, 2012; Couture et al., 2011; Hou et al., 2011; Li et al., 2011; Merle et al., 2011; Wang et al., 2011; Di Vona and Knauth, 2013; Hickner, 2013). One can mention ion exchange membranes, and a major application concerns separation membranes for electrochemical energy technologies, such as lithium polymer batteries and polymer electrolyte fuel cells.

Whereas polymer electrolytes for lithium batteries must work in absence of water to avoid corrosion and decomposition reactions, polymer electrolytes used in fuel cells and redox flow batteries contain significant amounts of water in hydrated nanometric domains that assure the proton or anion conduction inside a matrix made by the polymer backbones (Springer et al., 1991; Zawodzinski et al., 1993; Kreuer, 2001; Kreuer et al., 2004; Smitha et al., 2005; Diat and Gebel, 2008; Peckham et al., 2008; Hickner, 2012; Wu et al., 2013). Such polymers with a micro-phase separation between hydrated ionic conducting domains and electronically insulating polymer domains are also called ionomers.

Given the particular ion conduction mechanism, the water content of ionomers bears a particular importance (Zawodzinski et al., 1993; Gebel, 2000; Paddison, 2003; Mauritz and Moore, 2004; Majsztrik et al., 2007; Freger, 2009; Wu et al., 2011, 2013; Kumar et al., 2013; Yan et al., 2013). It fosters on one hand the ionic conductivity, but reduces on the other hand the mechanical and dimensional stability of the ionomer, because the high dielectric constant of water diminishes the Van der Waals interactions between macromolecular chains that determine the elastic properties of the ionomer (Alberti et al., 2008). The loss of dimensional stability is detrimental for the application in electrochemical energy technologies, because the swelling or shrinkage observed during changes of hydration might lead to delamination of metal electrodes in contact with the ionomers. This is particularly true in the case of fuel cells, where transients between high and low humidity conditions are observed during on-off cycles.

Water-uptake measurements of an ionomer are thus among the most important characterizations to be performed; the determination of the ionic conductivity as function of the hydration of the ionomer is also fundamental (Casciola et al., 2006; Peckham et al., 2008; Knauth and Di Vona, 2012; Di Vona et al., 2013; Knauth et al., 2013).

It is therefore tempting to analyze the interdependence of hydration and ionic conductivity also by theoretical means and to predict these properties using simple concepts and analytical equations, if available (Knauth and Di Vona, 2012; Narducci et al., 2014).

The following text is an attempt to present recent achievements made by our group for the phenomenological description of hydration and ionic conductivity of ionomers and their relationship. We will take as practical examples sulfonated poly-etherether-ketone (SPEEK) and sulfonated polyphenylsulfone (SPPSU) for which numerous experimental properties have been published over the years (Bauer et al., 2000; Rikukawa and Sanui, 2000; Li et al., 2003; Robertson et al., 2003; Roziere and Jones, 2003; Xing et al., 2004; Reyna-Valencia et al., 2006; Di Vona et al., 2009, 2010; Sgreccia et al., 2010; Wu et al., 2011; Hou et al., 2012; Knauth et al., 2013) but the discussed relations are also valid for other ionomers. 


\section{HYDRATION PROPERTIES}

The hydration equilibrium of an ionomer is governed by the Gibbs free energy of hydration that contains several contributions (Choi et al., 2005; Freger, 2009; Knauth et al., 2014; Narducci et al., 2014):

$\Delta G_{\text {hydration }}=\Delta G_{\text {osmotic }}+\Delta G_{\text {elastic }}+\Delta G_{\text {interface }}+\Delta G_{\text {electrostatic }}$

The driving force for the hydration is the osmotic pressure difference of water between the membrane and the environment ( $\Delta G_{\text {osmotic }}$ ). The osmotic pressure $\pi$ is related to the concentration of the electrolyte solution inside the ionomer and the outside water activity a $\left(\mathrm{H}_{2} \mathrm{O}\right)$ (given for example by the relative humidity $\mathrm{RH})$ :

$$
\pi=\frac{R T}{V_{0}} \ln \left(\frac{a\left(\mathrm{H}_{2} \mathrm{O}\right)}{x_{0, \mathrm{el}}}\right)
$$

In this equation, $x_{0, \mathrm{el}}$ is the water molar fraction in the electrolytic solution; $R$ is the gas constant, $T$ the absolute temperature, and $V_{0}$ the molar volume of water.

The osmotic driving force is counterbalanced by the elastic forces $\left(\Delta G_{\text {elastic }}\right)$ that hold the polymer together and which can be expressed by the elastic modulus $E$ of the ionomer. In the elastic domain of the ionomer, a linear relation exists between the deformation pressure $P$ (taken as equal to the osmotic pressure) and the volume strain (Lazare et al., 1956):

$$
P=\frac{V_{\text {wet }}-V_{\text {dry }}}{V_{\text {dry }}} E
$$

This relation is the tridimensional equivalent of the onedimensional Hooke's law for elastic solids.

The wet volume $V_{\text {wet }}$ is the sum of the volumes of the internal electrolyte solution and of the polymer backbone, which contains the fixed sulfonate anions and is considered incompressible. The dry volume $V_{\text {dry }}$ is the sum of the polymer backbone volume and the accessible open volume between macromolecular chains, called the "free volume" $V_{\text {free }}$. This free volume can be estimated using Bondi's equation (Bondi, 1964). In most polymers, it is considered to be between 10 and $25 \%$ of the dry molar volume.

Other contributions to the Gibbs free energy of hydration can be taken into account, including an interfacial energy term ( $\left.\Delta G_{\text {interface }}\right)$, due to the interface curvature of the hydrated domains inside the ionomer (Choi et al., 2005; Freger, 2009) and an electrostatic term $\left(\Delta G_{\text {electrostatic }}\right)$, due to the presence of electrically charged groups inside the ionomer (Lazare et al., 1956; Eikerling and Berg, 2011). These contributions are, however, more difficult to estimate. The interfacial term depends on the shape of the hydrophilic domains. In general, these are assumed to have a cylindrical or spherical shape but this depends on the amount of water present and the type of polymer (Gebel, 2000; Diat and Gebel, 2008). In absence of a complete analysis, the interface term, although reputedly related to the Schröder paradox (Freger, 2009), is thus difficult to assess. The electrostatic term, which can be written analytically only with the simplified linearized PoissonBoltzmann equation (Kortüm, 1965; Hamann et al., 2007), can be described in general only numerically, as done for instance in the early work by Gregor and coworkers (Lazare et al., 1956). These two terms are thus difficult to express in an analytical form and hard to predict.

We use in the following a description of the hydration of ionomers based on the equivalence between the osmotic pressure term and the elastic term. We thus assume that the osmotic pressure $\pi$ and the deformation pressure $P$ are identical.

The volume of the inner electrolyte solution can be written as the sum of the molar volumes of water and of protons present in the electrolyte. The latter being negligible, we can write:

$$
V_{\mathrm{el}}=\sum_{i} n_{i} V_{i} \approx n_{0} V_{0}
$$

A linear relationship can be postulated between $V_{\mathrm{el}}$ and the thermodynamic osmotic pressure $\pi$ (Gregor, 1951):

$$
V_{\mathrm{el}}=a \pi+b
$$

The parameters $a$ and $b$ depend upon the specific ionomer considered. Using the previous considerations, we can identify in Eq. 5:

$$
a=\frac{V_{\mathrm{dry}}}{E} \text { and } b=V_{\text {free }}
$$

We will in the following call $a$ "deformation parameter" and $b$ "free volume parameter."

A change of the mole number of water $n_{0}$ changes $V_{\mathrm{el}}$ in Eq. 4, but also in Eq. 5 by a modification of the osmotic pressure $\pi$ in Eq. 2 . The equilibrium water-uptake $n_{0 \text {,eq }}$ can then be found graphically or numerically: it is the simultaneous solution of Eqs 4 and 5 . As we use consistently molar quantities, $n_{0, \text { eq }}$ is directly equal to the hydration number $\lambda$, also called the water-uptake coefficient, defined as:

$$
n_{0, \mathrm{eq}}=\lambda=\frac{n\left(\mathrm{H}_{2} \mathrm{O}\right)}{n\left(\mathrm{SO}_{3} \mathrm{H}\right)}=\frac{\mathrm{WU}}{\mathrm{IEC} \times \mathrm{M}\left(\mathrm{H}_{2} \mathrm{O}\right)}
$$

The water-uptake WU is obtained from the polymer mass in wet and dry conditions. The ion exchange capacity IEC is expressed in $\mathrm{mol} / \mathrm{g}$ and $\mathrm{M}\left(\mathrm{H}_{2} \mathrm{O}\right)$ is the molar mass of water in $\mathrm{g} / \mathrm{mol}$. Using Eqs 4-6, we can give an analytical expression for the water-uptake coefficient:

$$
\lambda \approx \frac{V_{\text {dry }}}{E \cdot V_{0}} \pi+\frac{V_{\text {free }}}{V_{0}}
$$

In principle, the advantage of this approach is that it allows the prediction of water-uptake data based on only two physically relevant parameters: the deformation parameter, inversely proportional to the elastic modulus of the ionomer, and the free volume parameter, which is related to the available open space and can be estimated using Bondi's equation. The model assumes thermodynamic ideality, i.e., all activity coefficients are set to unity and partial volumes identified to the molar volume.

We will now check the reliability of the model prediction by confronting them with experimental hydration isotherms for 
SPEEK without cross-links and with some reticulation (Knauth et al., 2014). In this case, the deformation parameters were determined from the experimental elastic modulus $(\sim 1.1 \mathrm{GPa}$ for cross-linked and $\sim 0.6 \mathrm{GPa}$ for uncross-linked SPEEK) and its dry volume $(0.275 \mathrm{~L} / \mathrm{mol})$ giving values of $\mathrm{a}=7.5 \times 10^{-5}$ and $1.5 \times 10^{-4} \mathrm{~L} /(\mathrm{kg} . \mathrm{mol})$, respectively. The free volume parameter can be estimated from Bondi's equation using tabulated atomic or group contributions (Bondi, 1964) giving $b=0.13 \mathrm{~L} / \mathrm{mol}$.

Figure 1 shows experimental hydration isotherms and modeling based on the analytical equations for SPEEK with and without cross-linking sulfone bridges. The overall agreement between experiments and simulation is quite good, given that no fitting parameters were used, but only two physically meaningful constants.

We can recognize that the curves of cross-linked and uncrosslinked ionomers do intersect at a relative humidity value around $40 \%$. At higher humidity, the cross-linked ionomer shows a lower water uptake, as expected. However, more surprisingly, the hydration at lower humidity is higher for the cross-linked than for the uncross-linked ionomer. This is in fact a general finding observed for many other ionomers, see for example (Di Vona et al., 2009, 2010). Actually, the XL sample does not take up more water at low $\mathrm{RH}$, but it loses less. In cross-linked ionomers, the deformation is permanently fixed and conformational changes observed during hydration are impossible; there are, thus, lower changes in hydration number between high and low humidity. In other words, the ionomer with the higher stiffness is less sensible to changes of relative humidity.

Figure 2 shows water-uptake data for sulfonated polyphenylsulfone (SPPSU) with high degree of sulfonation (IEC $=3.56 \mathrm{eq} / \mathrm{kg}$ ) and after a cross-linking treatment (IEC $=2.35 \mathrm{eq} / \mathrm{kg}$, corresponding to a degree of crosslinking $\sim 0.7)$. The calculation was done with a deformation parameter $2 \times 10^{-4} \mathrm{~L} /$ (mol.bar) (SPPSU) and $10^{-4} \mathrm{~L} /$ (mol.bar) (XL-SPPSU) and a constant free volume parameter of $0.1 \mathrm{~L} / \mathrm{mol}$, which can be obtained from Bondi's equation.

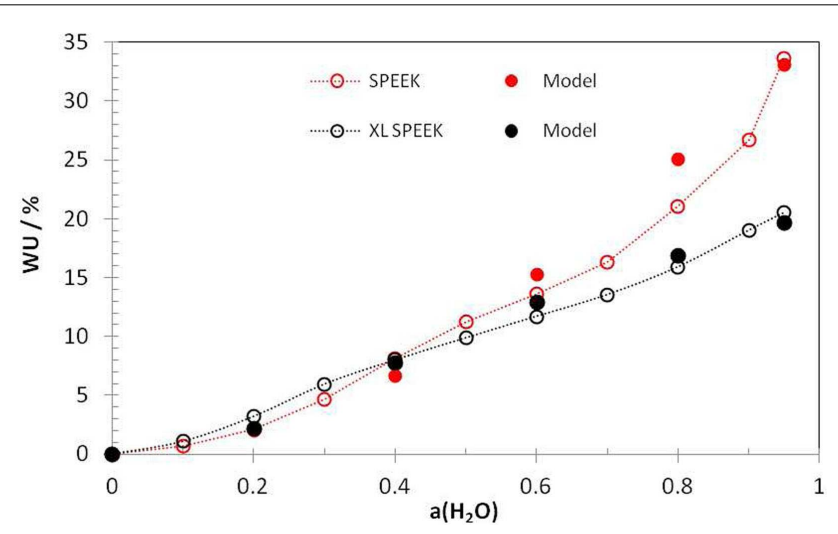

FIGURE 1 | Experimental water-uptake isotherms (open symbols) for uncross-linked (IEC $=2.5 \mathrm{eq} / \mathbf{k g}$ ) and cross-linked SPEEK $(X L$, degree of cross-linking $=0.45$, IEC $=1.5 \mathrm{eq} / \mathbf{k g}$ ) and model results using Eqs 2, 4, and $\mathbf{5}$ (full symbols). The deformation parameter was taken as $1.5 \times 10^{-4}$ (SPEEK) or $7.5 \times 10^{-5}$ (XL SPEEK) L $\mathrm{mol}^{-1} \mathrm{bar}^{-1}$ and the free volume parameter as $0.13 \mathrm{~L} / \mathrm{mol}$ in both cases. From Knauth et al. (2014).
Again, the change made by XL is mainly the reduced deformation parameter due to the larger elastic modulus. The agreement between model and experiment is again quite good, at high humidity especially for the XL ionomer. This is probably related to the fact that XL ionomers have a larger stiffness so that they are better described by an elastic model.

In order to verify that hypothesis, let us check also the water uptake in liquid water and the existence of a Schröder paradox in some of our samples, i.e., differences between the water-uptake measured in liquid water and that in saturated water vapor, which should be identical according to equilibrium thermodynamics. Several explanations for the Schröder paradox have been brought forward over the years, including slow changes of conformation of the ionomers [kinetic effect (Alberti et al., 2008)] or the interface curvature of hydrated domains [thermodynamic effect (Freger, 2009)]. Figure 3 shows a comparison of water uptake at high
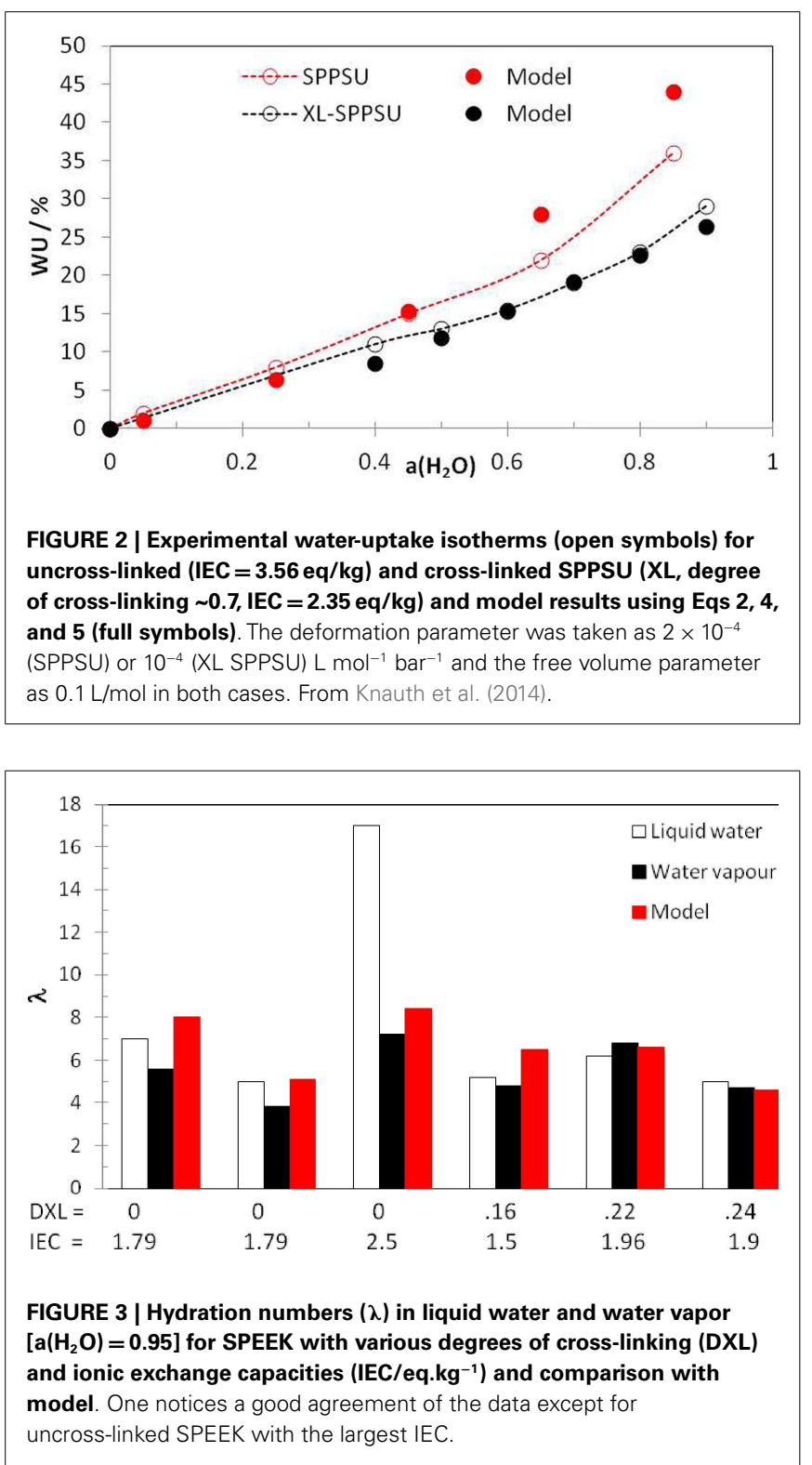
humidity, water uptake in liquid water and model data for SPEEK ionomers with various IEC and degrees of cross-linking. One can clearly observe that the Schröder paradox is only apparent for the highest IEC $=2.5 \mathrm{eq} / \mathrm{kg}$. XL ionomers do not show significant differences between water uptake in water vapor or liquid water and the model data are in good agreement. This seems to indicate that the appearance of a Schröder paradox is related to an ionomer with low stiffness and to irreversible plastic deformation of the ionomer in water, whereas in stiffer ionomers with high elastic modulus only elastic deformation is observed, which is well described by our elastic model.

Given that the durability of ionomers decreases with repeated swelling-drying cycles, a lower difference between water uptakes at high and low humidity should reduce the degradation issues for example under fuel-cell operation conditions, especially in electric vehicles with frequent on-off cycles. In that sense, crosslinked ionomers appear particular appealing for this application (Knauth et al., 2014). More discussion of the model assumptions and predictions and more experimental and modeling data for other ionomers, including Nafion, can be found in the reference (Knauth et al., 2014).

\section{PROTON CONDUCTIVITY}

High proton conductivity is an even more indispensable property for an ionomer separation membrane. Given the particular conduction mechanism through hydrated nanometric domains, the hydration is of fundamental importance for high proton conduction. The relation between proton conductivity and hydration will be the topic of this part.

Let us first specify that the ionic exchange capacity (IEC) of the ionomer alone is not a pertinent parameter to analyze the proton conductivity, because it does not consider the water content of the ionomer. We will in the following establish the proton concentration as a more relevant parameter. The proton concentration $\mathrm{c}$ (in $\mathrm{mol} / \mathrm{L}$ per liter) is defined using the IEC of the ionomer and its water-uptake WU:

$$
c=\frac{\mathrm{IEC} \cdot d}{\mathrm{WU}}
$$

$d$ is the solution density, taken as $1 \mathrm{~kg} / \mathrm{L}$. The relation of the proton concentration $c$ with the hydration number $\lambda$ (also called water-uptake coefficient) can be written:

$$
\lambda=\frac{1000 \cdot d}{\mathrm{M}\left(\mathrm{H}_{2} \mathrm{O}\right) \cdot c}
$$

These equations assume a full dissociation of the acidic groups; although this is certainly correct for super-acids at high water content, deviations from full dissociation will occur at lower hydration and are reflected in a large change of the apparent proton mobility (see below).

The proton conductivity $\sigma$ can be written as function of the proton concentration $c$ :

$$
\sigma=F \cdot u \cdot c
$$

$u$ is the apparent proton mobility inside the nanometric hydrated channels, which can now be calculated using the measured proton conductivity and the measured (or calculated, see above) water uptake. Figure 4 shows the dependence of the apparent proton mobility on the proton concentration at 25 and $100^{\circ} \mathrm{C}$. The very strong mobility dependence is characteristic of an electrolyte, where the proton dissociation depends on the hydration. Similar curves can for example be found for acetic acid solutions (Hamann et al., 2007). The difference between values at 25 and $100^{\circ} \mathrm{C}$ decreases with decreasing concentration, simply because the activation energy decreases when the solution gets more diluted and the Grotthuss structural diffusion mechanism becomes dominant (Kreuer et al., 2004).

The very strong change of mobility observed in Figure 2 at $\sqrt{ } \mathrm{c} \approx 3$ (corresponding according to Eq. 10 to $\lambda \sim 6$ ) can be related to the percolation threshold of the hydrated channels. A similar percolation threshold has been found by molecular dynamics for SPEEK (Mahajan and Ganesan, 2010a,b). Near the percolation threshold, the concentration dependence of conductivity can be written using a "universal" power law as function of the polymer volume fraction $\varphi\left(\varphi_{\mathrm{p}}\right.$ corresponds to the percolation threshold):

$$
\sigma=\sigma_{0}\left(\varphi-\varphi_{p}\right)^{\alpha}
$$

The critical exponent $\alpha=2$ is typical for three-dimensional percolation (Clerc et al., 1990). This law explains well the experimental power law $u \sim c^{-3}$ observed above the percolation threshold, given that the concentration is inversely proportional to the polymer volume fraction.

The linear part of the semi-logarithmic mobility plot in Figure 4 can be extrapolated to $c=0$ (infinite dilution). The extrapolated value at $25^{\circ} \mathrm{C}$ is somewhat lower than the proton mobility in pure water $\left(\approx 3.6 \times 10^{-3} \mathrm{~cm}^{2} /\right.$ V.s (Kortüm, 1965)). This is due to the fact that the hydrated domains have only a reduced size (expressed by the "porosity") and are not straight, but tortuous, so that protons have to move a longer way to cross the membrane as compared to the membrane thickness. These two factors can be taken into account by two phenomenological parameters, called membrane porosity $\varepsilon$ and tortuosity $\tau$. The proton mobility can be rescaled using these two parameters:

$$
u=u_{0} \cdot \frac{\varepsilon}{\tau}
$$

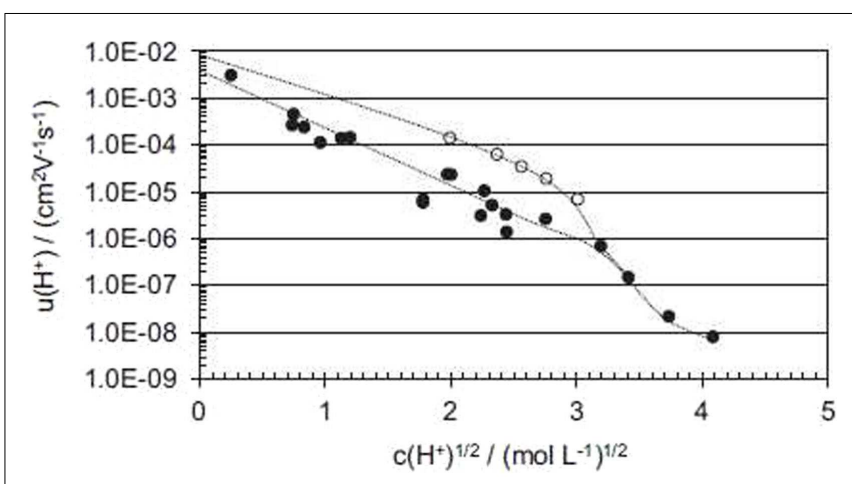

FIGURE 4 | Apparent proton mobility in SPEEK ionomers as function of the square root of the proton concentration (Di Vona et al., 2013). The open dots were determined at $100^{\circ} \mathrm{C}$, the closed dots at $25^{\circ} \mathrm{C}$. 


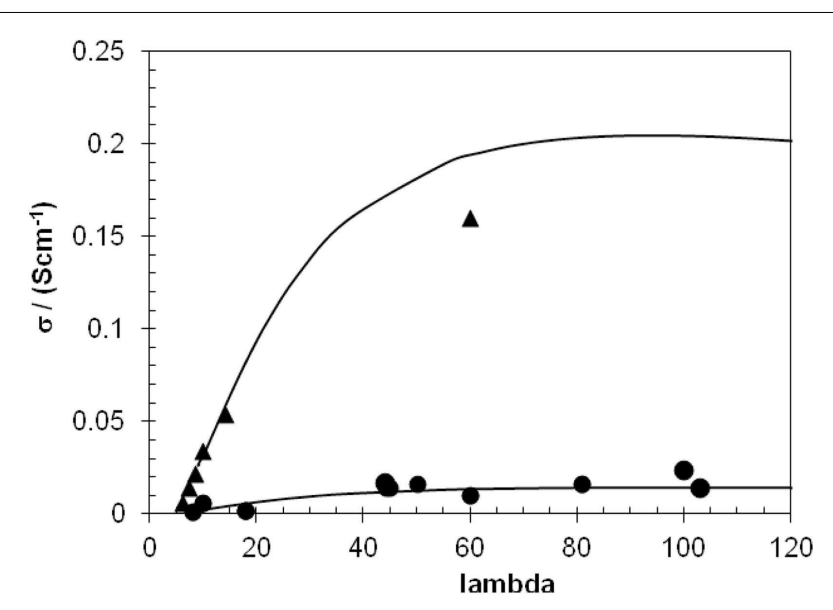

FIGURE 5 | Proton conductivity variation with the hydration number (a) at $25^{\circ} \mathrm{C}$ (dots), (b) at $100^{\circ} \mathrm{C}$ (triangles). An intermediate maximum of proton conductivity is observed for hydration numbers around 90 . At $100^{\circ} \mathrm{C}$, proton conductivity above $0.1 \mathrm{~S} / \mathrm{cm}$ can be obtained for hydration number 25 .

The ratio $\varepsilon / \tau \sim 0.2$ shows a relatively high tortuosity of SPEEK, as discussed previously in the literature (Mahajan and Ganesan, 2010a).

Using the mobility dependence on concentration, the maximum proton conductivity can be computed using Eq. 11: a maximum is expected at high hydration, because the mobility decreases strongly with concentration. Figure 5 shows this dependence for SPEEK at 25 and $100^{\circ} \mathrm{C}$; such a plot allows predicting the best conductivity achievable for an ionomer.

The maximum is observed for a quite high hydration number, about 90, and one may ask if such high values make sense as excessive swelling must be avoided to provide good durability. However, this plot allows also choosing conditions for combined high proton conductivity and acceptable swelling. For example, it allows predicting which hydration conditions are necessary for a desired area specific resistance. At $100^{\circ} \mathrm{C}$, proton conductivity above $0.1 \mathrm{~S} / \mathrm{cm}$, which is often considered the threshold to be reached by funding agencies, can be attained for a hydration number of 25 , which is more compatible with the requirements for low swelling. The excellent properties of XL-SPEEK membranes in fuel cells will be reported soon.

This plot allows also prediction of the area specific resistance of the ionomer under whatever hydration conditions. Given that the hydration itself can be valuably predicted using our simplified hydration model, the potential of a new ionomer can be evaluated based on only few experimental parameters: elastic modulus, dry molar volume, and proton conductivity at low (dry state) and high (in water) humidity.

\section{CONCLUSION}

We have presented a phenomenological model for the description of the hydration of ionomers. This model involves only two parameters, related to physical properties of the ionomer: the deformation parameter can be calculated from the elastic modulus and the dry volume of the ionomer. The free volume parameter can be estimated from the Bondi equation. Experimental and simulated hydration isotherms for SPEEK are in remarkable agreement; another strength of our simple model is that ionomer hydration properties can be predicted. The lower difference between hydration at low and high humidity for cross-linked ionomers should be an asset for higher durability under fuel-cell operation conditions (frequent on-off cycles).

The proton conductivity of ionomers depends very strongly on the hydration. Our approach based on the calculation of the apparent proton mobility, plotted vs. proton concentration, allows the determination of the percolation threshold and the ratio porosity/tortuosity of the ionomer membrane. Furthermore, optimal hydration conditions can be determined where good proton conductivity and acceptable swelling can be realized simultaneously in order to reach the required area specific resistance.

\section{REFERENCES}

Alberti, G., Narducci, R., and Sganappa, M. (2008). Effects of hydrothermal/thermal treatments on the water-uptake of Nafion membranes and relations with changes of conformation, counter-elastic force and tensile modulus of the matrix. J. Power Sources 178, 575-583. doi:10.1016/j.jpowsour.2007.09.034

Armand, M. (1994). The history of polymer electrolytes. Solid State Ionics. 69, 309-319. doi:10.1016/0167-2738(94)90419-7

Bauer, B., Jonesb, D. J., Rozièreb, J., Tchicayab, L., Albertic, G., Casciolac, M., et al. (2000). Electrochemical characterisation of sulfonated polyetherketone membranes. J. New Mater. Electrochem. Syst. 3, 93-98.

Bondi, A. (1964). van der Waals Volumes and Radii. J. Phys. Chem. 68, 441-451. doi:10.1021/j100785a001

Casciola, M., Alberti, G., Sganappa, M., and Narducci, R. (2006). On the decay of Nafion proton conductivity at high temperature and relative humidity. J. Power Sources 162, 141-145. doi:10.1016/j.jpowsour.2006.06.023

Choi, P., Jalani, N. H., and Datta, R. (2005). Thermodynamics and proton transport in Nafion - II. Proton diffusion mechanisms and conductivity. J. Electrochem. Soc. 152, E123-E130. doi:10.1149/1.1859814

Clerc, J. P., Giraud, G., Laugier, J. M., and Luck, J. M. (1990). The AC electricalconductivity of binary disordered-systems, percolation clusters, fractals and related models. Adv. Phys. 39, 191-308. doi:10.1080/00018739000101501

Couture, G., Alaaeddine, A., Boschet, F., and Ameduri, B. (2011). Polymeric materials as anion-exchange membranes for alkaline fuel cells. Prog. Polym. Sci. 36, 1521-1557. doi:10.1016/j.progpolymsci.2011.04.004

Di Vona, M. L., and Knauth, P. (2013). Sulfonated aromatic polymers as protonconducting solid electrolytes for fuel cells: a short review. Int. J. Res. Phys. Chem. Chem. Phys. 227, 595-614. doi:10.1524/zpch.2013.0337

Di Vona, M. L., Pasquini, L., Narducci, R., Pelzer, K., Donnadio, A., Casciola, M., et al. (2013). Cross-linked sulfonated aromatic ionomers via SO2 bridges: conductivity properties. J. Power Sources 243, 488-493. doi:10.1016/j.jpowsour.2013.05.127

Di Vona, M. L., Sgreccia, E., Licoccia, S., Alberti, G., Tortet, L., and Knauth, P. (2009). Analysis of temperature-promoted and solvent-assisted cross-linking in sulfonated poly(ether ether ketone) (speek) proton-conducting membranes. J. Phys. Chem. B 113, 7505-7512. doi:10.1021/jp9006679

Di Vona, M. L., Sgreccia, E., Tamilvanan, M., Khadhraoui, M., Chassigneux, C., Knauth, P., et al. (2010). High ionic exchange capacity polyphenylsulfone (SPPSU) and polyethersulfone (SPES) cross-linked by annealing treatment: Thermal stability, hydration level and mechanical properties. J. Memb. Sci. 354, 134-141. doi:10.1016/j.memsci.2010.02.058

Diat, O., and Gebel, G. (2008). Proton channels. Nat. Mater. 7, 13-14. doi:10.1038/ nmat2091

Eikerling, M. H., and Berg, P. (2011). Poroelectroelastic theory of water sorption and swelling in polymer electrolyte membranes. Soft Matter 7, 5976-5990. doi:10.1039/clsm05273j

Fergus, J. W. (2010). Ceramic and polymeric solid electrolytes for lithium-ion batteries. J. Power Sources 195, 4554-4569. doi:10.1016/j.jpowsour.2010.01.076

Fergus, J. W. (2012). Ion transport in sodium ion conducting solid electrolytes. Solid State Ionics 227, 102-112. doi:10.1016/j.ssi.2012.09.019

Freger, V. (2009). Hydration of ionomers and Schroeder's paradox in nafion. J. Phys. Chem. B 113, 24-36. doi:10.1021/jp806326a 
Gebel, G. (2000). Structural evolution of water swollen perfluorosulfonated ionomers from dry membrane to solution. Polymer 41, 5829-5838. doi:10.1016/ S0032-3861(99)00770-3

Gregor, H. P. (1951). GIBBS-Donnan equilibria in ion exchange resin systems. J. Am. Chem. Soc. 73, 642-650. doi:10.1021/ja01146a042

Hamann, C. H., Hamnett, A., and Vielstich, W. (2007). Electrochemistry, 2nd Edn. Weinheim: Wiley-VCH.

Hickner, M. A. (2012). Water-mediated transport in ion-containing polymers. J. Polymer. Sci. B Polymer. Phys. 50, 9-20. doi:10.1002/polb.22381

Hickner, M. A. (2013). Ion-containing polymers: new energy \& clean water. Mater. Today 13, 34-41. doi:10.1016/S1369-7021(10)70082-1

Hou, H. Y., Di Vona, M. L., and Knauth, P. (2011). Durability of sulfonated aromatic polymers for proton-exchange-membrane fuel cells. Chem. Sus. Chem. 4, 1526-1536. doi:10.1002/cssc.201000415

Hou, H. Y., Maranesi, B., Chailan, J.-F., Khadhraoui, M., Polini, R., Di Vona, M. L., et al. (2012). Crosslinked SPEEK membranes: mechanical, thermal, and hydrothermal properties. J. Mater. Res. 27, 1950-1957. doi:10.1557/jmr.2012.151

Knauth, P., and Di Vona, M. L. (2012). Sulfonated aromatic ionomers: analysis of proton conductivity and proton mobility. Solid State Ionics. 225, 255-259. doi:10.1016/j.ssi.2012.01.043

Knauth, P., Pasquini, L., Maranesi, B., Pelzer, K., Polini, R., and Di Vona, M. L. (2013). Proton mobility in sulfonated PolyEtherEtherKetone (SPEEK): influence of thermal crosslinking and annealing. Fuel Cells 13, 79-85. doi:10.1002/ fuce. 201200120

Knauth, P., Sgreccia, E., and Di Vona, M. L. (2014). Chemomechanics of acidic ionomers: hydration isotherms and physical model. J. Power Sources 267, 692-699. doi:10.1016/j.jpowsour.2014.05.127

Kortüm, G. (1965). Treatise on Electrochemistry, 2nd Edn. Amsterdam, NY: Elsevier.

Kreuer, K. D. (2001). On the development of proton conducting polymer membranes for hydrogen and methanol fuel cells. J. Memb. Sci. 185, 29-39. doi:10. 1016/S0376-7388(00)00632-3

Kreuer, K. D., Paddison, S. J., Spohr, E., and Schuster, M. (2004). Transport in proton conductors for fuel-cell applications: simulations, elementary reactions, and phenomenology. Chem. Rev. 104, 4637-4678. doi:10.1021/cr020715f

Kumar, M., Edwards, B. J., and Paddison, S. J. (2013). A macroscopic model of proton transport through the membrane-ionomer interface of a polymer electrolyte membrane fuel cell. J. Chem. Phys. 138, 13. doi:10.1063/1.4789960

Lazare, L., Sundheim, B. R., and Gregor, H. P. (1956). A model for cross-linked polyelectrolytes. J. Phys. Chem. 60, 641-648. doi:10.1021/j150539a035

Li, L., Zhang, J., and Wang, Y. X. (2003). Sulfonated poly(ether ether ketone) membranes for direct methanol fuel cell. J. Memb. Sci. 226, 159-167. doi:10.1016/j. memsci.2003.08.018

Li, X. F., Zhang, H. M., Mai, Z. S., and Zhang, H. Z. I (2011). Vankelecom, Ion exchange membranes for vanadium redox flow battery (VRB) applications. Energy Environ. Sci. 4, 1147-1160. doi:10.1039/c0ee00770f

Mahajan, C. V., and Ganesan, V. (2010a). Atomistic simulations of structure of solvated sulfonated poly(ether ether ketone) membranes and their comparisons to nafion: I. Nanophase segregation and hydrophilic domains. J. Phys. Chem. B 114, 8357-8366. doi:10.1021/jp104078h

Mahajan, C. V., and Ganesan, V. (2010b). Atomistic simulations of structure of solvated sulfonated poly(ether ether ketone) membranes and their comparisons to nafion: II. Structure and transport properties of water, hydronium ions, and methanol. J. Phys. Chem. B 114, 8367-8373. doi:10.1021/jp1040794

Majsztrik, P. W., Satterfield, M. B., Bocarsly, A. B., and Benziger, J. B. (2007). Water sorption, desorption and transport in Nafion membranes. J. Memb. Sci. 301, 93-106. doi:10.1021/jp7103243

Mauritz, K. A., and Moore, R. B. (2004). State of understanding of Nafion. Chem. Rev. 104, 4535-4585. doi:10.1021/cr0207123

Merle, G., Wessling, M., and Nijmeijer, K. (2011). Anion exchange membranes for alkaline fuel cells: a review. J. Memb. Sci. 377, 1-35. doi:10.1016/j.memsci.2011. 04.043

Meyer, W. H. (1998). Polymer electrolytes for lithium-ion batteries. Adv. Mater. Weinheim 10, 439. doi:10.1002/(SICI)1521-4095(199804)10:6

Narducci, R., Di Vona, M. L., and Knauth, P. (2014). Cation-conducting ionomers made by ion exchange of sulfonated poly-ether-ether-ketone: Hydration, mechanical and thermal properties and ionic conductivity. J. Memb. Sci. 465, 185-192. doi:10.1016/j.memsci.2014.03.073
Paddison, S. J. (2003). Proton conduction mechanisms at low degrees of hydration in sulfonic acid-based polymer electrolyte membranes. Ann. Rev. Mater. Res. 33, 289-319. doi:10.1146/annurev.matsci.33.022702.155102

Peckham, T. J., Schmeissert, J., and Holdcroft, S. (2008). Relationships of acid and water content to proton transport in statistically sulfonated proton exchange membranes: Variation of water content via control of relative humidity. J. Phys. Chem. B 112, 2848-2858. doi:10.1021/jp077218t

Reyna-Valencia, A., Kaliaguine, S., and Bousmina, M. (2006). Structural and mechanical characterization of poly(ether ether ketone) (PEEK) and sulfonated PEEK films: Effects of thermal history, sulfonation, and preparation conditions. J. Appl. Polymer. Sci. 99, 756-774. doi:10.1002/app.22551

Rikukawa, M., and Sanui, K. (2000). Proton-conducting polymer electrolyte membranes based on hydrocarbon polymers. Prog. Polym. Sci. 25, 1463-1502. doi:10.1016/S0079-6700(00)00032-0

Robertson, G. P., Mikhailenko, S. D., Wang, K., Xing, P., Guiver, M. D., et al. (2003). Casting solvent interactions with sulfonated poly(ether ether ketone) during proton exchange membrane fabrication. J. Memb. Sci. 219, 113-121. doi:10.1016/S0376-7388(03)00193-5

Roziere, J., and Jones, D. J. (2003). Non-fluorinated polymer materials for proton exchange membrane fuel cells. Ann. Rev. Mater. Res. 33, 503-555. doi:10.1146/ annurev.matsci.33.022702.154657

Sgreccia, E., Chailan, J. F., Khadhraoui, M., Di Vona, M. L., and Knauth, P. (2010). Mechanical properties of proton-conducting sulfonated aromatic polymer membranes: stress-strain tests and dynamical analysis. J. Power Sources 195, 7770-7775. doi:10.1016/j.jpowsour.2009.09.061

Smitha, B., Sridhar, S., and Khan, A. A. (2005). Solid polymer electrolyte membranes for fuel cell applications - a review. J. Memb. Sci. 259, 10-26. doi:10.1016 j.memsci.2005.01.035

Springer, T. E., Zawodzinski, T. A., and Gottesfeld, S. (1991). Polymer electrolyte fuel-cell model. J. Electrochem. Soc. 138, 2334-2342. doi:10.1149/1.2085971

Wang, Y., Chen, K. S., Mishler, J., Cho, S. C., and Adroher, X. C. (2011). A review of polymer electrolyte membrane fuel cells: technology, applications, and needs on fundamental research. Appl. Energy 88, 981-1007. doi:10.1016/j.apenergy.2010. 09.030

Wu, L., Zhang, Z., Ran, J., Zhou, D., Li, C., and Xu, T. (2013). Advances in protonexchange membranes for fuel cells: an overview on proton conductive channels (PCCs). Phys. Chem. Chem. Phys. 15, 4870-4887. doi:10.1039/c3cp50296a

Wu, X. M., Wang, X. W., He, G. H., and Benziger, J. (2011). Differences in water sorption and proton conductivity between nafion and SPEEK. J. Polymer Sci. B Polymer. Phys. 49, 1437-1445. doi:10.1002/polb.22326

Xing, P. X., Robertson, G. P., Guiver, M. D., Mikhailenko, S. D., Wang, K., and Kaliaguine, S. (2004). Synthesis and characterization of sulfonated poly(ether ether ketone) for proton exchange membranes. J. Memb. Sci. 229, 95-106. doi:10.1016/j.memsci.2003.09.019

Yan, X. M., He, G. H., Wu, X. M., and Benziger, J. (2013). Ion and water transport in functionalized PEEK membranes. J. Memb. Sci. 429, 13-22. doi:10.1016/j. memsci.2012.11.026

Zawodzinski, T. A., Springer, T., Davey, J., Jestel, R., Lopez, C., Valerio, J., et al. (1993) A comparative-study of water-uptake by and transport through ionomeric fuelcell membranes. J. Electrochem. Soc. 140, 1981-1985. doi:10.1149/1.2220749

Conflict of Interest Statement: The authors declare that the research was conducted in the absence of any commercial or financial relationships that could be construed as a potential conflict of interest.

Received: 06 July 2014; accepted: 20 October 2014; published online: 06 November 2014.

Citation: Knauth P and Di Vona ML (2014) Hydration and proton conductivity of ionomers: the model case of sulfonated aromatic polymers. Front. Energy Res. 2:50. doi: $10.3389 /$ fenrg.2014.00050

This article was submitted to Fuel Cells, a section of the journal Frontiers in Energy Research.

Copyright $(0) 2014$ Knauth and Di Vona. This is an open-access article distributed under the terms of the Creative Commons Attribution License (CC BY). The use, distribution or reproduction in other forums is permitted, provided the original author (s) or licensor are credited and that the original publication in this journal is cited, in accordance with accepted academic practice. No use, distribution or reproduction is permitted which does not comply with these terms. 